\title{
Serum erythropoietin level predicts the prognosis of chronic heart failure with or without anemia
}

\author{
LING GUO ${ }^{1}$, AI-HONG WANG ${ }^{1}$, YONG-LE SUN ${ }^{1}$, LIN LV $^{1}$ and CHONG-EN XU ${ }^{2}$ \\ Departments of ${ }^{1}$ Cardiology and ${ }^{2}$ Cardiac Surgery, Provincial Hospital Affiliated to Shandong University, Jinan, \\ Shandong 250021, P.R China
}

Received May 15, 2013; Accepted September 2, 2013

DOI: $10.3892 /$ etm.2013.1307

\begin{abstract}
The aim of this study was to explore the correlation of erythropoietin (EPO) with N-terminal pro-B-type natriuretic peptide (NT-proBNP) and high sensitivity C-reactive protein (hs-CRP) in patients with chronic heart failure (CHF) or $\mathrm{CHF}$ complicated with anemia, in addition to its correlation with the prognosis of the patient. A total of $217 \mathrm{CHF}$ patients were enrolled in this study. The patients were graded according to the cardiac function criteria of the New York Heart Association (NYHA). The serum EPO, NT-proBNP and hs-CRP levels of the patients were determined. The patients were followed up for $\geq 24$ months. The EPO expression level in patients with NYHA II-IV CHF was significantly higher compared with that in the control group $(\mathrm{P}<0.05)$. EPO expression increased with the aggravation of $\mathrm{CHF}$, exhibiting significant differences amongst the various NYHA graded groups $(\mathrm{P}<0.05)$. The EPO expression level increased significantly with an increase in NHA grade in addition to the severity of the anemia in the patients with $\mathrm{CHF}$ complicated by anemia $(\mathrm{P}<0.05)$. In the patients who succumbed (mortality group), the expression level of EPO was significantly higher and the hemoglobin level was significantly lower compared with those of the survival group $(\mathrm{P}<0.05)$. The EPO expression levels were elevated in $\mathrm{CHF}$ patients and patients with $\mathrm{CHF}$ and anemia. The level of expression correlated positively with the severity of $\mathrm{CHF}$ as well as that of anemia. Serum EPO measurements were successful in predicting the mortality and re-hospitalization rates of $\mathrm{CHF}$ patients at the end point, within two years of follow-up.
\end{abstract}

Correspondence to: Professor Chong-En $\mathrm{Xu}$, Department of Cardiac Surgery, Provincial Hospital Affiliated to Shandong University, 324 Jingwu-Weiqi Road, Jinan, Shandong 250021, P.R. China

E-mail: xjspcn@163.com

Key words: heart failure, erythropoietin, N-terminal pro-B-type natriuretic peptide, high sensitivity $\mathrm{C}$-reactive protein, prognosis

\section{Introduction}

The treatment of chronic heart failure (CHF) is currently an area of interest in the field of cardiovascular disease. Numerous factors such as neurohormonal activation, inflammation and oxidative stress may lead to a poor prognosis of CHF and a one-year mortality rate as high as $30-40 \%(1,2)$. Although drug treatments for CHF have improved markedly over the last decade, $\sim 20 \%$ of patients require regular in-hospital treatments every year due to recurrent attacks, a consequence of which is that the disease develops into a refractory condition and is ultimately fatal. Currently, a high number of patients with $\mathrm{CHF}$ are awaiting medical treatment. For these patients, finding correctable curative factors and then curing or relieving related symptoms is vital; among these factors is anemia $(3,4)$. Anemia has an incidence rate of between 40 and $50 \%$ in patients with $\mathrm{CHF}$, which increases with the increased severity of CHF (5-7). The incidence rate may be as high as $80 \%$ in patients with heart function NYHA IV. Anemia is an independent risk factor amongst the various factors that induce CHF-related mortalities and has the potential to double the mortality rate $(8,9)$. Anemia-associated histanoxia causes vasodilatation, which activates the sympathetic nervous system, leading to peripheral vasoconstriction. Kidney vasoconstriction subsequently activates the renin-angiotensin-aldosterone system which further aggravates kidney and peripheral vasoconstriction due to an increase in angiotensin II; in the long-term this is harmful for the heart $(10,11)$. However, the significance of anemia in the treatment and deterioration of $\mathrm{CHF}$ has been neglected. Furthermore, anemia aggravates the symptoms of CHF and makes its treatment increasingly difficult. Anemia in patients with CHF is primarily caused by a deficiency of erythropoietin (EPO) or the decreased sensitivity of the spinal cord to EPO, and the application of EPO in the treatment of CHF complicated by anemia may have a marked curative effect (12-15).

Neurohormonal and inflammatory activation may trigger $\mathrm{CHF}$ and scholars have long sought serological markers capable of reflecting the effectiveness of CHF treatment and predicting its long-term prognosis (16-18). The severity of anemia positively correlates with that of $\mathrm{CHF}$; a higher grade of $\mathrm{CHF}$, according to the cardiac function standard of the New York Heart Association (NYHA), indicates lower hemoglobin ( $\mathrm{Hb})$ levels, which indicates a poorer prognosis $(5,19,20)$. EPO levels 
in the blood may predict the mortality and hospitalization risk for $\mathrm{CHF}$ patients in the years following treatment.

EPO, which is produced by the kidneys, promotes the proliferation and differentiation of erythroid progenitor cells in the spinal cord. Recently, with a deepened understanding of its action mechanisms and its potential actions as a multifunctional cytokine, EPO has attracted an increasing amount of attention. EPO has anti-apoptotic and anti-oxidative properties and promotes the activation of endothelial precursor cells for angiogenesis. Due to its multiple properties, EPO has been assumed to be a stress hormone; EPO is highly expressed under aggravated CHF conditions (21-23). A close correlation between EPO and CHF has previously been demonstrated. However, since the overexpression of EPO may also be induced by other stimuli related to tissue hypoperfusion, including oxidative stress and the subsequent production of oxygen radicals, the predictive value of EPO for the prognosis of CHF requires verification.

Therefore, in the current study, the EPO levels of patients with $\mathrm{CHF}$ were determined in order to explore the correlation between EPO expression and the severity of $\mathrm{CHF}$ and $\mathrm{CHF}$ complicated with anemia. The results were analyzed further using known neurohormonal and inflammatory activation markers used for CHF prognostic prediction. The findings of this study may provide theoretical references for the early intervention and prognostic prediction of $\mathrm{CHF}$.

\section{Subjects and methods}

Subjects. A total of $217 \mathrm{CHF}$ patients who received treatment at Shandong Provincial Hospital (Jinan, China) between January 2007 and December 2010 were recruited for this study. All patients met the Framingham diagnostic criteria of CHF (3) and were graded II-IV, according to the cardiac function standard of the NYHA. In total, 69 patients were diagnosed with accompanied anemia (Hb concentration, <110 g/l).

The study was conducted in accordance with the Declaration of Helsinki and approved by the Ethics Committee of Shandong Provincial Hospital. Written informed consent was obtained from all participants.

Exclusion criteria. Patients who met any of the following criteria were excluded from the study: age, $<18$ years; currently pregnant; diagnosed with a malignant tumor; presented with a systemic infection; exhibited gastrointestinal bleeding; and had undergone a cerebral vascular accident, major surgery, or recombinant EPO treatment within the previous three months.

Medical history. The patients received clinical examinations and were subsequently NYHA-graded. All patients were consulted further, every 1-3 months or dependent on the pathogenic condition. The control group comprised 50 non- $\mathrm{CHF}$ patients who were admitted to hospital during the same period exhibiting symptoms of palpitations and chest pain. No significant differences in age or gender were observed between the groups.

Follow-up. The end point of this study was the time to mortality due to any cause or re-hospitalization due to CHF. All patients were followed-up for 24 months.
Table I. Clinical features and medications of $217 \mathrm{CHF}$ patients.

A. Clinical features

\begin{tabular}{lc}
\hline Features & Patient number \\
\hline Age (years; mean \pm SE) & $69.7 \pm 10.4$ \\
Males & $145(67.0 \%)$ \\
Left ventricular ejection fraction & \\
(mean \pm SD) & $36 \pm 12.6$ \\
Hyperlipidemia & $118(54.3 \%)$ \\
Anemia & $69(31.8 \%)$ \\
Kidney failure & $85(39.2 \%)$ \\
Smoking & $87(40.1 \%)$ \\
Hypertension & $131(60.4 \%)$ \\
Diabetes & $74(34.1 \%)$ \\
Ischemic heart disease & $160(73.7 \%)$ \\
Chronic atrial fibrillation & $66(30.4 \%)$ \\
Transient ischemic attack/ & \\
cerebrovascular accident & $25(11.5 \%)$ \\
Percutaneous transluminal coronary & \\
angioplasty or coronary artery & \\
bypass surgery & $51(23.5 \%)$ \\
\hline
\end{tabular}

B. Medication

\begin{tabular}{lr}
\hline Drugs & Patient number \\
\hline ACE inhibitors/ARBs & $156(71.9 \%)$ \\
$\beta$-blockers & $135(62.2 \%)$ \\
Spironolactone & $83(38.2 \%)$ \\
Diuretics & $151(69.6 \%)$ \\
Digoxin & $55(25.3 \%)$ \\
\hline
\end{tabular}

CHF, chronic heart failure; ACE, angiotensin-converting enzyme; ARBs, angiotensin receptor blockers.

EPO determination. Fasting ulnar venous blood $(3 \mathrm{ml})$ was extracted in the morning for all participants and subsequently centrifuged at $730 \mathrm{x}$ g for $10 \mathrm{~min}$. The serum was separated and stored at $-20^{\circ} \mathrm{C}$. Serum EPO levels were determined using an radioimmunoassay.

$\mathrm{Hb}, \mathrm{N}$-terminal pro-B-type natriuretic peptide (NT-proBNP) and high sensitivity $C$-reactive protein ( $h s-C R P)$ analysis. $\mathrm{Hb}$ content was determined using a Coulter ${ }^{\circledR}$ full-automatic hematology analyzer (Beckman-Coulter, Miami, FL, USA) using the cyan-methemoglobin method. The serum level of NT-proBNP was determined by ELISA. hs-CRP levels were determined using a latex particle-enhanced turbidometric immunoassay.

Statistical analysis. Data were analyzed using SPSS 10.0 software (SPSS, Inc., Chicago, IL, USA). Enumeration data were presented as the mean $\pm \mathrm{SE}$. The Student's t-test was performed for pairwise comparisons and one-factor ANOVA 
Table II. NYHA grading and EPO, NT-proBNP and hs-CRP levels upon hospital admission.

\begin{tabular}{lcccc}
\hline NYHA grading & Cases & EPO $(\mathrm{mU} / \mathrm{ml})$ & NT-proBNP $(\mathrm{pg} / \mathrm{ml})$ & $\mathrm{hs}-\mathrm{CRP}(\mathrm{mg} / \mathrm{l})$ \\
\hline Control & 50 & $2.36 \pm 0.73$ & $149.27 \pm 68.31$ & $1.60 \pm 0.41$ \\
NYHA II & 74 & $14.10 \pm 1.63$ & $512.34 \pm 107.31$ & $2.40 \pm 0.32$ \\
NYHA III & 96 & $26.70 \pm 2.94$ & $2217.00 \pm 294.35$ & $3.60 \pm 0.54$ \\
NYHA IV & 47 & $35.90 \pm 5.81$ & $5951.00 \pm 424.62$ & $4.50 \pm 0.48$ \\
P-value & & $<0.01$ & $<0.01$ & $<0.05$ \\
\hline
\end{tabular}

NYHA, New York Heart Association; EPO, erythropoietin; NT-proBNP, N-terminal pro-B-type natriuretic peptide; hs-CRP, high sensitivity C-reactive protein.

was performed in order to compare the EPO levels among groups with different NYHA grades. $\mathrm{P}<0.05$ was considered to indicate a statistically significant difference.

\section{Results}

General patient data. The mean age of the $217 \mathrm{CHF}$ patients was $69.7 \pm 10.4$ years. According to the NYHA criteria, 74 patients $(34.1 \%)$ were grade II, $96(44.2 \%)$ were grade III, and 47 were grade IV (21.7\%). Of all the patients, 69 (31.8\%) had CHF accompanied by anemia. The clinical features and medications of the patients are summarized in Table I, and comparisons of the EPO, NT-proBNP and hs-CRP levels among different NYHA graded groups are summarized in Table II.

Correlation of EPO with $\mathrm{Hb}$ and NYHA grading in $\mathrm{CHF}$ patients with anemia. In total, 69 of the 217 patients were diagnosed with CHF accompanied by anemia, which corresponded to an incidence of $31.8 \%$. The EPO level increased with an increase in the NYHA grade in addition to the severity of anemia, demonstrating significant differences among the various groups $(\mathrm{P}<0.05)$. The results are summarized in Table III.

Comparisons between the EPO and Hb levels of the survival and mortality groups. All patients were followed-up through phone calls, clinical visits or hospitalized medical records. During the follow-up period, 34 of the 217 patients succumbed, and 183 survived with an average follow-up time of $3.5 \pm 1.3$ years. The EPO expression level of the mortality group was significantly higher, compared with that of the survival group, whereas the $\mathrm{Hb}$ level was significantly lower, $(\mathrm{P}<0.05)$. The results are summarized in Table IV.

Correlation of EPO, NT-proBNP and hs-CRP with patient prognosis. Of the 217 patients, 34 (15.6\%) succumbed, 67 (30.9\%) were re-hospitalized due to CHF and 42 (19.4\%) were hospitalized due to severe CHF. The mean levels of EPO, NT-proBNP, and hs-CRP at the various end points are summarized in Tables $\mathrm{V}$ and VI. The results indicate that the EPO level is relatively successful in predicting the mortality and re-hospitalization rates of CHF patients at end points within two years of follow-up $(\mathrm{P}<0.01)$, and NT-proBNP levels may also be used as a successful predictor.
Table III. Correlations of EPO and Hb with NYHA grading in patients with $\mathrm{CHF}$ complicated with anemia.

\begin{tabular}{lccc}
\hline Groups & Cases & EPO $(\mathrm{mU} / \mathrm{ml})$ & $\mathrm{Hb}(\mathrm{g} / \mathrm{dl})$ \\
\hline NYHA II & 9 & $14.65 \pm 4.53^{\mathrm{a}}$ & $13.16 \pm 2.19^{\mathrm{a}}$ \\
NYHA III & 28 & $27.94 \pm 8.46^{\mathrm{a}}$ & $11.68 \pm 2.03^{\mathrm{a}}$ \\
NYHA IV & 32 & $38.47 \pm 11.83^{\mathrm{a}}$ & $10.27 \pm 1.94^{\mathrm{a}}$ \\
P-value & & $<0.05$ & $<0.05$
\end{tabular}

Values are provided as the mean $\pm \mathrm{SD}$. ${ }^{\mathrm{a}} \mathrm{P}<0.05$, compared with the next higher NYHA grade. NYHA, New York Heart Association; EPO, erythropoietin; $\mathrm{CHF}$, chronic heart failure; $\mathrm{Hb}$, hemoglobin.

Table IV. Comparisons between the EPO and Hb levels of the survival and mortality groups.

\begin{tabular}{lccc}
\hline Groups & Cases & EPO $(\mathrm{mU} / \mathrm{ml})$ & $\mathrm{Hb}(\mathrm{g} / \mathrm{dl})$ \\
\hline Survival & 183 & $17.48 \pm 5.29$ & $12.64 \pm 2.26$ \\
Mortality & 34 & $36.74 \pm 9.77^{\mathrm{a}}$ & $10.56 \pm 2.03^{\mathrm{a}}$ \\
\hline
\end{tabular}

${ }^{\mathrm{a}} \mathrm{P}<0.05$, compared with the survival group. EPO, erythropoietin; $\mathrm{Hb}$, hemoglobin.

\section{Discussion}

In this study, the serum EPO levels of patients with CHF of various NYHA grades were determined, and compared with those of non-CHF patients who were recruited as controls. The results show that the EPO levels in patients with NYHA II-IV $\mathrm{CHF}$ are significantly higher than those in observed in non-CHF patients $(\mathrm{P}<0.05)$. In addition, EPO levels significantly increase with an increase in NYHA grade $(\mathrm{P}<0.05)$. Furthermore, this study demonstrates that with an increase in NYHA grade, $\mathrm{Hb}$ levels decrease significantly. These results demonstrate that EPO is important in the development of CHF. As anoxia is a vital regulatory factor of EPO, patients with $\mathrm{CHF}$ presumably suffer from tissue hypoperfusion, which causes internal environmental hypoxia and subsequently leads to the overexpression of EPO. Alternatively, tissue hypoperfusion 
Table V. Comparisons between the EPO, NT-proBNP and hs-CRP levels of surviving patients and patients who succumbed to CHF.

\begin{tabular}{lcccc}
\hline Event end point & Cases & EPO $(\mathrm{mU} / \mathrm{ml})$ & NT-proBNP $(\mathrm{pg} / \mathrm{ml})$ & hs-CRP $(\mathrm{mg} / \mathrm{l})$ \\
\hline Mortality & 34 & $36.74 \pm 9.77$ & $6451.00 \pm 467.82$ & $5.10 \pm 0.44$ \\
Survival & 183 & $17.48 \pm 5.29$ & $2493.00 \pm 329.43$ & $4.20 \pm 0.75$ \\
P-value & & $<0.001$ & $<0.001$ & 0.59 \\
\hline
\end{tabular}

EPO, erythropoietin; NT-proBNP, N-terminal pro-B-type natriuretic peptide; hs-CRP, high sensitivity C-reactive protein; CHF, chronic heart failure.

Table VI. Comparisons between the EPO, NT-proBNP and hs-CRP levels of re-hospitalized and re-hospitalization-free patients

\begin{tabular}{lcccc}
\hline Event end point & Cases & EPO (mU/ml) & NT-proBNP (pg/ml) & hs-CRP (mg/l) \\
\hline Mortality or re-hospitalization & 143 & $29.86 \pm 9.40$ & $3577.00 \pm 415.33$ & $4.30 \pm 0.73$ \\
Survival and hospitalization-free & 74 & $12.60 \pm 5.62$ & $1147.00 \pm 307.46$ & $3.70 \pm 0.61$ \\
P-value & & $<0.01$ & $<0.01$ & 0.34 \\
\hline
\end{tabular}

EPO, erythropoietin; NT-proBNP, N-terminal pro-B-type natriuretic peptide; hs-CRP, high sensitivity C-reactive protein.

may lead to other types of stimulation that upregulate the expression of EPO, such as oxidative stress leading to the production of oxygen radicals $(24,25)$.

Epidemiological data have indicated that being female, increased age, kidney insufficiency, low body weight and severe cardiac dysfunction are high risk factors for $\mathrm{CHF}$ complicated by anemia (26). The incidence of anemia increases with an increase in the severity of $\mathrm{CHF}$, and may achieve an incidence of $79.1 \%$ in patients with NYHA IV CHF. In this study, 69 patients $(31.8 \%)$ presented with CHF complicated by anemia, and their EPO levels increased significantly with the increased severity of $\mathrm{CHF}(\mathrm{P}<0.05)$. This finding indicates that EPO is expressed at elevated levels in patients with $\mathrm{CHF}$ complicated by anemia and correlates positively with the severity of CHF. Serum EPO is an efficient predictor of the mortality and re-hospitalization rates of $\mathrm{CHF}$ patients. The increased expression of serum EPO correlates with a poor $\mathrm{CHF}$ prognosis. Furthermore, EPO expression is independent of $\mathrm{Hb}$ and other markers that have been confirmed to indicate the severity of CHF.

In addition, this study reveals that serum EPO expression levels correlate with the severity of CHF predicted using NYHA grading. The two years of follow-up in this study further demonstrates that NT-ProBNP expression is an independent predictor of the mortality and re-hospitalization rates of patients with CHF. Furthermore, this study demonstrates that serum EPO expression may serve as a predictor in this respect, whereas hs-CRP levels do not successfully predict the mortality rate.

In conclusion, EPO expression is increased in patients with $\mathrm{CHF}$ and in those with $\mathrm{CHF}$ complicated by anemia, and the increase correlates with the severity of CHF. Thus, EPO levels may successfully predict the prognosis of CHF, and serum EPO expression performs an important role in the progression of CHF. Furthermore, EPO, NT-ProBNP and hs-CRP correlate with one another. These findings indicate that neurohormonal activation and inflammation are clinical responses to late $\mathrm{CHF}$. Therefore, the determination of serum EPO expression is clinically significant for the prediction of the development, outcome and prognosis of $\mathrm{CHF}$.

\section{Acknowledgements}

This study was supported by Shandong Provincial Natural Science Foundation, No.: Y2007C052.

\section{References}

1. Levy D, Kenchaiah S, Larson MG, et al: Long-term trends in the incidence of and survival with heart failure. N Engl J Med 347: 1397-1402, 2002.

2. Roger VL, Weston SA, Redfield MM, et al: Trends in heart failure incidence and survival in a community-based population. JAMA 292: 344-350, 2004.

3. Agarwal AK and Katz SD: Future directions in management of anemia in heart failure. Heart Fail Clin 6: 385-395, 2010.

4. Tang WH, Tong W, Jain A, et al: Evaluation and long-term prognosis of new-onset, transient, and persistent anemia in ambulatory patients with chronic heart failure. J Am Coll Cardiol 51: 569-576, 2008

5. Anand IS, Kuskowski MA, Rector TS, et al: Anemia and change in hemoglobin over time related to mortality and morbidity in patients with chronic heart failure: results from Val-HeFT. Circulation 112: 1121-1127, 2005.

6. O'Meara E, Clayton T, McEntegart MB, et al; CHARM Committees and Investigators: Clinical correlates and consequences of anemia in a broad spectrum of patients with heart failure: results of the Candesartan in Heart Failure: Assessment of Reduction in Mortality and Morbidity (CHARM) Program. Circulation 113: 986-994, 2006.

7. Young JB, Abraham WT, Albert NM, et al; OPTIMIZE-HF Investigators and Coordinators: Relation of low hemoglobin and anemia to morbidity and mortality in patients hospitalized with heart failure (insight from the OPTIMIZE-HF registry). Am J Cardiol 101: 223-230, 2008. 
8. Bansal N, Tighiouart H, Weiner D, et al: Anemia as a risk factor for kidney function decline in individuals with heart failure. Am J Cardiol 99: 1137-1142, 2007.

9. Felker GM, Adams KF Jr, Gattis WA and O'Connor CM: Anemia as a risk factor and therapeutic target in heart failure. J Am Coll Cardiol 44: 959-966, 2004.

10. Caramelo C, Justo S and Gil P: Anemia in heart failure: pathophysiology, pathogenesis, treatment, and incognitae. Rev Esp Cardiol 60: 848-860, 2007 (In Spanish).

11. Terrovitis JV, Anastasiou-Nana M, Kaldara E, Drakos SG, Nanas SN and Nanas JN: Anemia in heart failure: pathophysiologic insights and treatment options. Future Cardiol 5: 71-81, 2009.

12. Belonje AM, de Boer RA and Voors AA: Recombinant human Epo treatment: beneficial in chronic kidney disease, chronic heart failure, or both? Editorial to: "Correction of anemia with erythropoietin in chronic kidney disease (stage 3 or 4): effects on cardiac performance by Pappas et al.". Cardiovasc. Drugs Ther 22: 1-2, 2008.

13. Büyüklü M: Heart failure: erythropoietin in treatment of anemia Anadolu Kardiyol Derg 7: 342-343, 2007 (In Turkish).

14. Cohen RS, Karlin P, Yushak M, Mancini D and Maurer MS The effect of erythropoietin on exercise capacity, left ventricular remodeling, pressure-volume relationships, and quality of life in older patients with anemia and heart failure with preserved ejection fraction. Congest Heart Fail 16: 96-103, 2010.

15. Kotecha D, Ngo K, Walters JA, Manzano L, Palazzuoli A and Flather MD: Erythropoietin as a treatment of anemia in heart failure: systematic review of randomized trials. Am Heart J 161: 822-831, 2011.

16. Balion C, Santaguida PL, Hill S, et al: Testing for BNP and NT-proBNP in the diagnosis and prognosis of heart failure. Evid Rep Technol Assess (Full Rep) 142: 1-147, 2006

17. Tsutamoto T, Sakai H, Nishiyama K, et al: Direct comparison of transcardiac increase in brain natriuretic peptide (BNP) and $\mathrm{N}$-terminal proBNP and prognosis in patients with chronic heart failure. Circ J 71: 1873-1878, 2007.
18. Yu CM, Fung JW, Zhang Q, et al: Improvement of serum NT-ProBNP predicts improvement in cardiac function and favorable prognosis after cardiac resynchronization therapy for heart failure. J Card Fail 11: S42-46, 2005.

19. Lupón J, Urrutia A, González B, et al: Prognostic significance of hemoglobin levels in patients with heart failure. Rev Esp Cardiol 58: 48-53, 2005 (In Spanish).

20. Varadarajan P, Gandhi S, Sharma S, Umakanthan B and Pai RG: Prognostic significance of hemoglobin level in patients with congestive heart failure and normal ejection fraction. Clin Cardiol 29: 444-449, 2006.

21. Akram K and Pearlman BL: Congestive heart failure-related anemia and a role for erythropoietin. Int J Cardiol 117: 296-305, 2007.

22. Ruifrok WP, Qian C, Silljé HH, et al: Heart failure-associated anemia: bone marrow dysfunction and response to erythropoietin. J Mol Med (Berl) 89: 377-387, 2011.

23. TimmerSA,De Boer K, Knaapen P, Götte MJ and Van Rossum AC: The potential role of erythropoietin in chronic heart failure: from the correction of anemia to improved perfusion and reduced apoptosis? J Card Fail 15: 353-361, 2009.

24. Ogino A, Takemura G, Kawasaki M, et al: Erythropoietin receptor signaling mitigates renal dysfunction-associated heart failure by mechanisms unrelated to relief of anemia. J Am Coll Cardiol 56: 1949-1958, 2010.

25. Palazzuoli A, Silverberg D, Iovine F, et al: Erythropoietin improves anemia exercise tolerance and renal function and reduces B-type natriuretic peptide and hospitalization in patients with heart failure and anemia. Am Heart J 152: 1096. e9-15, 2006.

26. Tang WH and Yeo PS: Epidemiology of anemia in heart failure. Heart Fail Clin 6: 271-278, 2010. 